\title{
Parametric Linear Modeling of Circular cMUT Membranes in Vacuum
}

\author{
Hayrettin Köymen, Senior Member, IEEE, Muhammed N. Şenlik, Student Member, IEEE, \\ Abdullah Atalar, Fellow, IEEE, and Selim Olcum, Student Member, IEEE
}

\begin{abstract}
We present a lumped element parametric model for the clamped circular membrane of a capacitive micromachined ultrasonic transducer (cMUT). The model incorporates an electrical port and two sets of acoustic ports, through which the cMUT couples to the medium. The modeling approach is based on matching a lumped element model and the mechanical impedance of the cMUT membrane at the resonance frequencies in vacuum. Very good agreement between finite element simulation results and model impedance is obtained. Equivalent circuit model parameters can be found from material properties and membrane dimensions without a need for finite element simulation.
\end{abstract}

\section{INTRODUCTION}

$\mathrm{P}$ IEZOELECTRIC and piezomagnetic transducers are very successfully modeled by means of both distributed equivalent networks and lumped element circuits. The research and results obtained over fifty years make reliable and accurate designs possible. A very good account of the history of model development for these devices is given in [1].

Capacitive ultrasonic transducers, on the other hand, followed a different route. These transducers have been in existence for a longer period. Mason derived an acoustical impedance expression for an unbiased membrane of such a transducer in 1942, when operated in vacuum [2]. Similarly, the dynamic behavior of an unbiased transducer at low frequencies in vacuum was modeled by a lumped element equivalent circuit many years ago [2], [3]. Such modeling is very useful for single capacitive microphones with very thin membranes and for airborne applications only, because air presents very light acoustic loading. Capacitive micro-machined ultrasonic transducers (cMUTs), which belong to this class, emerged in the last decade as a result of developments in microfabrication technology [4], [5]. The very promising potential of these devices attracted attention to the modeling of these devices under different acoustic loading conditions.

The developments in the modeling of cMUTs can be categorized into three types: (a) mathematical modeling, (b) modeling with simulations, and (c) modeling with

Manuscript received September 26, 2006; accepted January 8, 2007. This work was supported in part by TUBITAK under project grant $105 \mathrm{E} 023$.

The authors are with the Electrical and Electronics Engineering Department, Bilkent University, Ankara, 06800 Turkey (e-mail: koymen@ee.bilkent.edu.tr).

Digital Object Identifier 10.1109/TUFFC.2007.376 equivalent circuits. Both electrical and mechanical modeling are employed in these approaches. Most of the work done up to now includes the modeling and optimization of a single device [6], [7] or an array of cMUTs [8]-[13] in immersion medium with finite element simulations, experimental work, and theoretical approaches. In a typical approach to obtain an equivalent circuit model, the starting point is the solution of the differential equation that describes the membrane motion [2] and the calculation of the mechanical impedance of the membrane [5], [14], [15]. In this approach, using this impedance in series with the spring-softening negative capacitance and the radiation impedance of an equal-sized piston forms the equivalent circuit. It is shown in [16] and [17] that including the mechanical impedance of the membrane obtained in vacuum into the equivalent circuit directly is not sufficient to model the cMUT properly, especially for immersion devices. It is possible to find the membrane shape under bias analytically [18], but mechanical impedance of the membrane cannot be calculated under nonuniform force distribution when biased or with partial electrode coverage. In this case, a finite element (FEM) simulation is required that can be done with cMUT-specific packages [9], [19]-[21] or with a commercially available software package [22]-[25]. Moreover, these simulations can be used to predict the loss mechanisms [26], or predict induced effects such as bending stress in the membranes [27].

FEM simulations provide a very good infrastructure for testing designs. There is a need for the guidance provided by an accurate equivalent circuit to design cMUTs. A common practice is to employ an equivalent circuit generally referred to as Mason's equivalent circuit or the same equivalent circuit with Mason's impedance expression replaced by a series $L C$ section [2], [5], [14], [15]. These equivalent circuits predict both the mechanical and electrical domain small signal operations at frequencies lower than the resonance frequency reasonably. It is possible to obtain the equivalent circuit parameters from measurement [28] or from simulations. Equivalent circuits can also include the loss mechanisms, such as energy coupling to membrane supports, which can be observed in FEM simulations [29]. Modeling approaches are employed to improve the electrical termination for better performance [30] or for better electromechanical coupling [31].

cMUTs are typically used immersed in water. Immersion cannot change the mechanical structure of the membrane but interaction between the immersion medium and the membrane affects the dynamics of the membrane. Our 
principle aim is to derive an accurate lumped element model for a cMUT cell, which can subsequently be used for cells in arrays. We aim to model for the radiation impedance of individual cells when immersed in liquid, coupling among the cells, and radiation impedance of the array, similarly, using the parametric model for a single cell. In order to achieve this goal, the following effects must be modeled and combined:

- Mechanics of the membrane, excluding the effect of electromechanical coupling,

- The electromechanical transformer ratio,

- Coupling between cells in an array,

- Radiation impedance of an array of cMUT cells immersed in liquid,

- The nonlinear behavior of cMUT when it is driven by large amplitude signals.

This kind of model enables one to design cMUT arrays without using FEM simulations. Using such a model makes it possible to employ very powerful techniques offered by circuit theory in design and analysis, as in the case of piezoelectric transducers. In this paper, we present an equivalent circuit model for membrane mechanics, which is the first step toward a comprehensive parametric model.

Behavior of the membrane in vacuum is determined only by the mechanical structure and material properties of the membrane. In other words, vacuum is the most suitable medium to characterize the mechanics of the membrane, since it isolates the effects induced by the immersion. We present a parametric modeling approach to produce a lumped element model for a cMUT membrane, where model parameters are derived from its operational characteristics in vacuum only. It is shown that the parameters in the model have fixed numerical values when stripped from material properties, which we call normalized circuit element values. We show that the proposed approach and the model describe the linear operation of a cMUT membrane very accurately under different operational conditions.

We use the Mason's impedance expression [2] for a membrane without any bias as the starting point. A normalization procedure is proposed which yields a dimensionless impedance expression defined over a dimensionless frequency range. This allows us to determine the model parameters in a normalized form, which are independent of material properties. It is shown that these parameter values are the same for all membranes made of any material used in cMUT production and for all possible membrane dimensions. These dimensionless parameters are then denormalized to model the particular cMUT membrane.

Mason's impedance expression models thin membranes, where radius-to-thickness ratio is very large, quite accurately. When the membranes are thick, their dynamic behavior deviates from the predictions of this model. We show that the same approach can be extended to membrane dimensions that violate the explicit and implicit assumptions in Mason's impedance expression and to membranes that are biased in a way similar to that used in
cMUT operation. We performed FEM simulations for unbiased membranes with radius-to-thickness ratios ranging from 5 to 80, and obtained normalized model parameters as a function of this ratio only. Then we considered the effect of bias on the model parameters of similar membranes. We simulated biased membranes and show that few model parameters are affected by bias. We present accurate expressions to represent this effect in terms of bias voltage and gap height-to-thickness ratio.

\section{Parametric Modeling of cMUTs in Vacuum}

\section{A. Mason's Impedance Expression for Clamped Thin Membranes}

The theory of operation of capacitive transducers has been known for a century. The mechanical behavior of clamped thin membranes in vacuum is well studied. The mechanical impedance, defined as the ratio of the pressure to the volume velocity, of such a membrane when the membrane is driven by a uniformly distributed force over its surface has been given by Mason [2]. The ratio of total force to average velocity, which is volume velocity divided by the surface area, is a more useful impedance definition from a measurement and simulation point of view, and is equal to the Mason's impedance multiplied by the square of the surface area. Accuracy of this impedance expression is also verified by experiments [5], [15] and by simulation $[22]$. The impedance, $j X m(\omega)=$ (total force) $/$ (average velocity), is given below in a normalized form:

$$
X_{m}(\omega)=\left(S \rho l_{t} \frac{\sqrt{c}}{a^{2}}\right) x_{1} x_{2} \frac{N\left(x_{1}, x_{2}\right)}{D\left(x_{1}, x_{2}\right)}
$$

with

$$
\begin{aligned}
N\left(x_{1}, x_{2}\right)= & x_{1} x_{2}\left[x_{2} J_{0}\left(x_{1}\right) I_{1}\left(x_{2}\right)+x_{1} J_{1}\left(x_{1}\right) I_{0}\left(x_{2}\right)\right], \\
D\left(x_{1}, x_{2}\right)= & x_{1} x_{2}\left[x_{2} J_{0}\left(x_{1}\right) I_{1}\left(x_{2}\right)+x_{1} J_{1}\left(x_{1}\right) I_{0}\left(x_{2}\right)\right] \\
& -2\left(x_{1}^{2}+x_{2}^{2}\right) J_{1}\left(x_{1}\right) I_{1}\left(x_{2}\right),
\end{aligned}
$$

where $a$ is the radius, $S$ is area $\pi a^{2}, \rho$ is the density of the membrane material, $l_{t}$ is the thickness, and hence $S \rho l_{t}$ is the mass of the membrane. The arguments are $x_{1}=k_{1} a$ and $x_{2}=k_{2} a$, and are interrelated as

$$
x_{2}^{2}=x_{1}^{2}+a^{2}(d / c)
$$

where

$$
\begin{aligned}
k_{1} & =\sqrt{\frac{\sqrt{d^{2}+4 c \omega^{2}}-d}{2 c}}, k_{2}=\sqrt{\frac{\sqrt{d^{2}+4 c \omega^{2}}+d}{2 c}}, \\
c & =Y_{o} \frac{\left(1+T / Y_{o}\right) l_{t}^{2}}{12 \rho\left(1-\sigma^{2}\right)}, \text { and } d=\frac{T}{\rho} .
\end{aligned}
$$

$Y_{o}$ is Young's modulus, $\sigma$ is the Poisson's ratio of the material, and $T$ is the residual stress. 
The angular frequency is

$$
\omega=\sqrt{c} k_{1} k_{2}=\sqrt{c} \frac{x_{1} x_{2}}{a^{2}}=\frac{\sqrt{c}}{a^{2}} \omega_{n},
$$

where $\omega_{n}=x_{1} x_{2}$ is the normalized angular frequency. Hence

$$
a^{2} \frac{d}{c}=\frac{T}{Y_{o}}\left(\frac{a}{l_{t}}\right)^{2} \frac{12\left(1-\sigma^{2}\right)}{1+T / Y_{o}} .
$$

When there is a residual stress in the membrane, the two frequency parameters, $x_{1}$ and $x_{2}$, are related through $a^{2} d / c$, which is a function of residual stress, material properties, and the radius-to-thickness ratio, $a / l_{t}$.

Let $j X_{n}(\omega)$ denote the normalized impedance as

$$
X_{n}(\omega)=\frac{X_{m}(\omega)}{S \rho l_{t} \sqrt{c} / a^{2}}=x_{1} x_{2} \frac{N\left(x_{1}, x_{2}\right)}{D\left(x_{1}, x_{2}\right)} .
$$

When written in this form, the normalized impedance expression is independent of membrane dimensions and material properties in the absence of residual stress in the membrane. This expression is dependent only on the circular geometry of the clamped membrane. The zeroes and poles of this equation describe the resonances and antiresonances of a circular membrane in terms of normalized angular frequency, $\omega_{n}$, which is also independent of material properties and dimensions.

Transduction elements, which operate in thickness mode or any other extensional mode, also have a distributed nature, and are very successfully modeled by sections of transmission lines, whether they are bulk- or shearwave devices [1]. While extensional mode devices lend themselves readily to transmission line models, vibrating membranes do not. Unlike extensional mode devices, clamped membrane dimensions (radius or thickness) do not change during vibration, despite the fact that the surface area changes. The resonances of this structure follow a sequence predicted by the impedance in (1), which is significantly different from what transmission lines can predict. The mechanical resonance frequencies are not equally spaced. Such a resonance behavior describes a modal structure, which can better be modeled by a lumped element circuit, rather than transmission lines.

In this work we adopted a lumped element approach to produce a parametric model for a clamped membrane vibrating in vacuum. It is shown that a circuit model of the form given in Fig. 1 represents the mechanical dynamics of the membrane exactly.

In order to determine the model parameters of Fig. 1, it is sufficient to match the model impedance and (6) at the first series resonance frequency and at as many parallel resonance frequencies as required [32]. As far as the model order is concerned, we need a resonating $L C$ (inductorcapacitor or mass-spring) section for each of the first series resonance and successive parallel resonances. In other words, we must maintain

$$
X_{i n}\left(\omega_{s 1}\right)=X_{n}\left(\omega_{s 1}\right)=0
$$

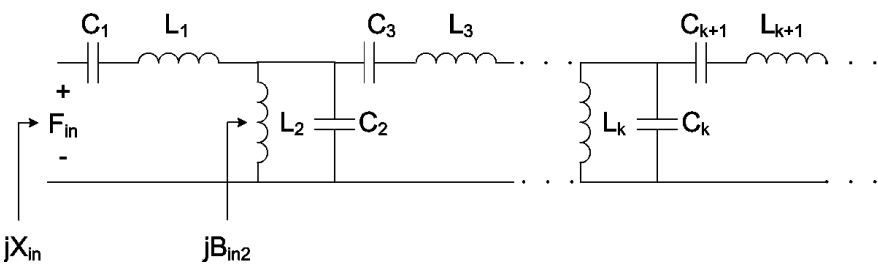

Fig. 1. Lumped element model of mechanical dynamics of the membrane.

and

$$
\left.\frac{d X_{i n}}{d \omega_{n}}\right|_{\omega_{s 1}}=\left.\frac{d X_{n}}{d \omega_{n}}\right|_{\omega_{s 1}}=\beta
$$

where $\omega_{s 1}$ is the normalized angular frequency of the first series resonance. We also have

$$
B_{i n 2}\left(\omega_{p i}\right)=B_{n}\left(\omega_{p i}\right)=0 \quad i=1,2, \ldots,
$$

and

$$
\left.\frac{d B_{i n 2}}{d \omega_{n}}\right|_{\omega_{p i}}=\left.\frac{d B_{n}}{d \omega_{n}}\right|_{\omega_{p i}}=\xi_{i} \quad i=1,2, \ldots
$$

where $B_{n}\left(\omega_{n}\right)=-1 / X_{n}\left(\omega_{n}\right)$, and $\omega_{p i}$ are the normalized parallel angular resonance frequencies $\omega_{p 1}, \omega_{p 2}$, etc., for successive parallel resonances.

We note that the actual membrane reactance $X_{m}$ and susceptance $B_{m}$ can be found from their normalized counterparts using (6). Their respective derivatives are related to the derivatives of $X_{m}$ and $B_{m}$ as

$$
\frac{d X_{n}\left(\omega_{n}\right)}{d \omega_{n}}=\frac{d X_{m}(\omega)}{d \omega} / S \rho l_{t}
$$

and

$$
\frac{d B_{n}\left(\omega_{n}\right)}{d \omega_{n}}=\frac{S \rho l_{t}}{a^{4} / c} \frac{d B_{m}(\omega)}{d \omega}
$$

\section{B. Model of a Clamped Thin Membrane Without Any Residual Stress}

$X_{i n}\left(\omega_{s 1}\right), B_{i n 2}\left(\omega_{p i}\right)$, and their derivatives at these frequencies are rational polynomial functions of the radial frequency, where the polynomial coefficients are functions of the model parameters, $L_{1}, C_{1}, L_{2}, \ldots$, of the circuit in Fig. 1. Model parameters must be determined such that (7) to (10) are satisfied.

If the first $i$ many parallel resonances are considered, the set of equations given in (7) to (10) provides $2(i+$ 1 ) independent equations. We can determine $L_{1}, C_{1}, L_{2}$, $C_{2}, \ldots, L_{k}, C_{k}$, where $k=i+1$, uniquely using these equations. The details of this procedure are described in Appendix A. The model parameters for $k=1$ and $k=4$ are given in Fig. 2 .

There is excellent agreement between the impedance of this model and the impedance expression in a frequency range up to the largest parallel resonance frequency used 




$1^{\text {st }}$ Order; no parallel circuit $C_{l}=0.005089$ $L_{I}=1.883$

(a)

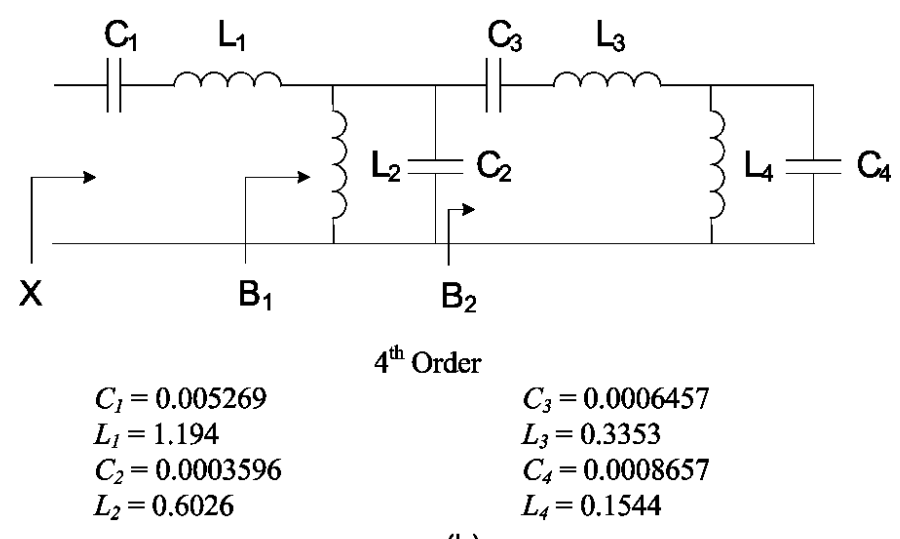

(b)

Fig. 2. First and fourth-order models with normalized element values.

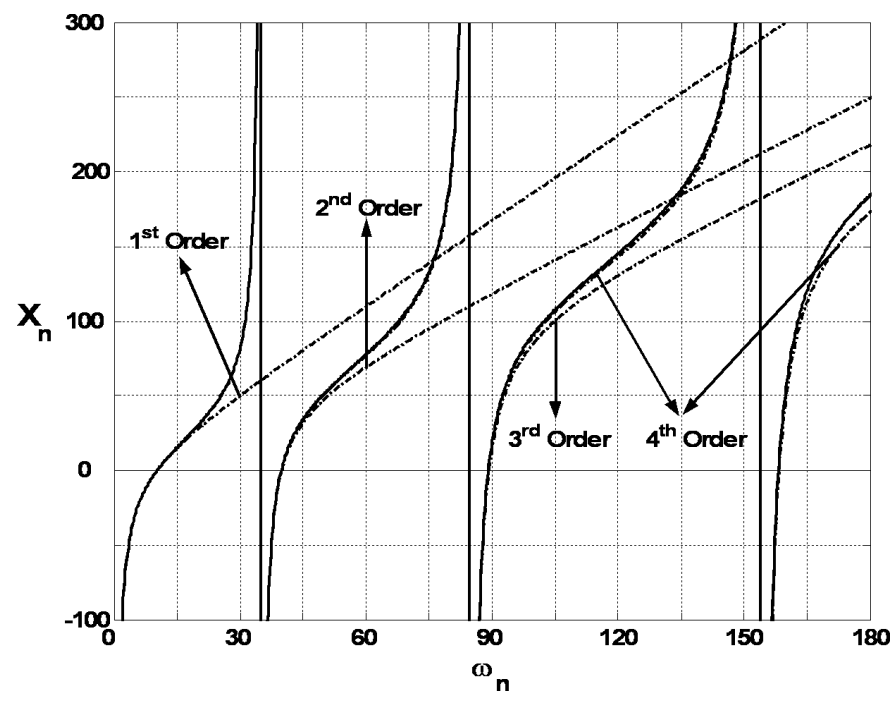

Fig. 3. Comparison of the agreement of the impedance for first, second, third, and fourth-order models and Mason's impedance expression.

in the modeling. A comparison of the agreement of the model impedance and the normalized Mason's impedance expression is given in Fig. 3. The rms error calculated up to the first parallel resonance frequency is $58 \%$ for the first-order model and $0.002 \%$ for the fourth-order model. A similar agreement is also obtained in thin membranes, such as the one with an $a / l_{t}$ ratio of 80 .

The fourth-order model represents the membrane behavior very accurately over the frequency range up to the third parallel resonance.

As the model order is increased, the inductive element $L_{1}$ decreases and $L_{2}$ increases, while $L_{1}+L_{2}$ remain al- most constant at about 1.8. This value is the approximate series inductance in [2], effective in the vicinity of series resonance. $L_{1}+L_{2}$ contain the mass of the membrane, which corresponds to a normalized inductance of 1 . All other inductive elements are pseudo masses, and are present because of the fact that the membrane is clamped and vibrating.

This is in agreement with the way the impedance is defined in Mason's expression. Only the average value of the particle velocity distribution across the membrane surface is considered when calculating the impedance. However, the deviations from the average velocity also represent an energy content taken away from the driver, and even have resonances. This impedance definition means that we are basically driving the membrane disc, and any other mechanical activity, such as wrinkles on the surface, takes its energy from this driving force. Inductances other than the membrane mass contained in $L_{1}+L_{2}$ represent the motional elements of this activity.

It is important to note here that the normalized model parameters are valid for a thin membrane as long as it is circular and clamped. The denormalized parameters, $L_{m 1}$, $C_{m 1}, L_{m 2}, C_{m 2}, \ldots, L_{m k}, C_{m k}$, etc., of a particular membrane can be obtained using the following relations:

$$
L_{m k}=\left(S \rho l_{t}\right) L_{k} \quad k=1,2,3, \ldots
$$

and

$$
C_{m k}=\frac{a^{4} / c}{S \rho l_{t}} C_{k}=\frac{a^{2}}{l_{t}^{3}} \frac{1}{\pi \rho\left(c / l_{t}^{2}\right)} C_{k} .
$$

\section{Thick Membranes}

A fundamental assumption in Mason's impedance expression is that the membrane is very thin, or, in other words, the radius-to-thickness ratio, $a / l_{t}$ is very large compared to unity. The effect of the membrane thickness on the stiffness is only implicit in the coefficient "c." The performance of this model can be checked by comparing the impedance found for the model to the impedance obtained by simulating cMUT membranes of increasing $a / l_{t}$ ratio in vacuum.

We simulated silicon nitride membranes with a radius $a=20 \mu \mathrm{m}$ and $a / l_{t}$ ratio of $80,40,20$, and 10 in vacuum using the finite element method (ANSYS; ANSYS, Inc., Canonsburg, PA). The membrane is driven by a uniform force distribution, and impedance of the membrane is evaluated as the ratio of total force to the average particle velocity on the surface. We observed very good agreement when $a / l_{t}=80$. However, as the membrane gets thicker, a discrepancy between the model predictions and simulation results emerges. The fourth-order model impedance is plotted in Fig. 4 together with simulation results for $a / l_{t}=10$, where the model impedance is significantly different. It is clear that the thicker membranes vibrate in a different manner compared to a thin membrane, even when they are unbiased. 




Fig. 4. Comparison of fourth-order model of Fig. 2(b) with finite element simulation results for $a / l_{t}=10$.

TABLE I

Coefficients of Generating Polynomials for a Fourth-ORder MODEL.

\begin{tabular}{ccccc}
\hline & $q_{3}$ & $q_{2}$ & $q_{1}$ & $q_{0}$ \\
\hline$C_{1}$ & 0.0006836 & 0.03329 & -0.0002277 & 0.005197 \\
$L_{1}$ & -6.523 & -3.868 & 0.02025 & 1.202 \\
$C_{2}$ & -0.05052 & 0.01738 & -0.0001251 & 0.0003291 \\
$L_{2}$ & 8.256 & 0.9559 & 0.002023 & 0.5991 \\
$C_{3}$ & -0.004007 & 0.003106 & -0.00005532 & 0.0006432 \\
$L_{3}$ & -5.921 & 10.49 & 0.02132 & 0.3082 \\
$C_{4}$ & -0.05627 & 0.03320 & -0.0001758 & 0.0008004 \\
$L_{4}$ & 6.112 & -0.4836 & 0.01525 & 0.1541 \\
\hline
\end{tabular}

The methodology developed above is valid for thick membranes also. Using the same model morphology of Fig. 1, normalized model parameters are recalculated using values obtained from the FEM results for $a / l_{t}=5,10$, 20,40 , and 80 , in the same way as in Section II-B. For different $a / l_{t}$, we found the following cubic polynomials for model parameters, which approximate them up to the third significant figure.

$$
\left\{\begin{array}{l}
L_{k} \\
C_{k}
\end{array}\right\}=q_{3}\left(\frac{l_{t}}{a}\right)^{3}+q_{2}\left(\frac{l_{t}}{a}\right)^{2}+q_{1}\left(\frac{l_{t}}{a}\right)+q_{0} \text { for } \frac{l_{t}}{a} \leq 0.2 .
$$

The polynomial coefficients $q_{i}$ for a fourth-order model are given in Table I.

We compared the finite element simulation results with the model impedance for different $a / l_{t}$ values. There is excellent agreement for $a / l_{t}=20$ for frequencies up to the third parallel resonance frequency. Fig. 5 shows the performance of the model for a thicker membrane $\left(a / l_{t}=5\right)$, where a good agreement up to the second parallel resonance frequency is observed.

We also studied the dependence of the normalized model parameters to the variation of material properties

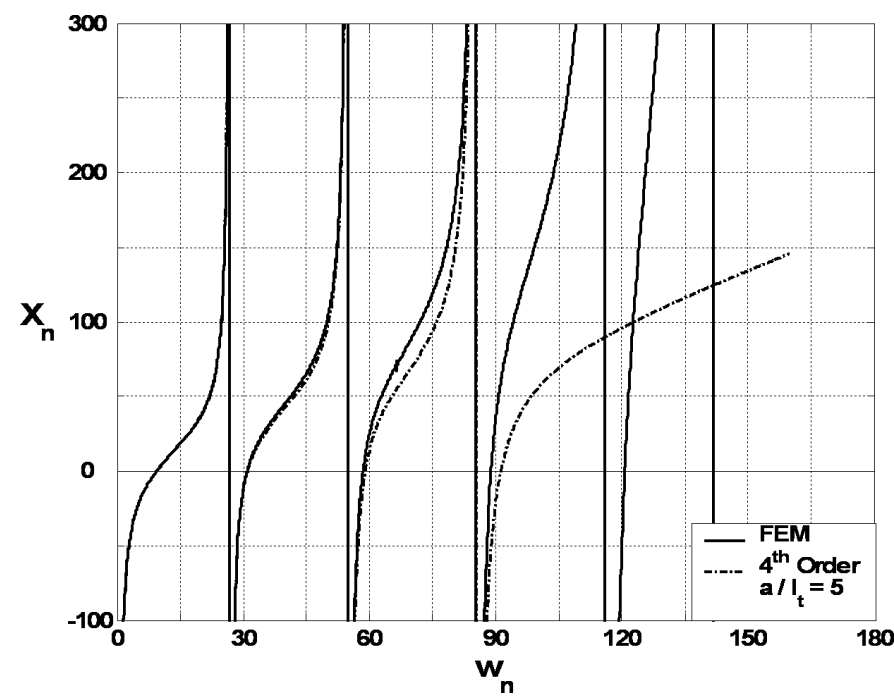

Fig. 5. Fourth-order model impedances, as obtained from Table I, for a thick membrane compared with finite element simulation.

in thick membranes. A membrane with an $a / l_{t}$ ratio of 20 but made of three different materials were simulated. Materials were silicon, silicon nitride, and silicon carbide, which is comparatively stiffer. All normalized parameters obtained for the model are similar to that of silicon nitride within $0.1 \%$, which shows that normalization given here strips the normalized model of material properties in thick membranes also.

In line with Mason's assumptions, a circular symmetry in the membrane and in the driving conditions is assumed. The circular cMUT membranes conform to this assumption and two-dimensional models are adequate for simulations. On the other hand, asymmetric modes cannot appear under this assumption. Excitation of asymmetric modes is not expected since we consider isolated single membranes and there is no loading on the membrane in vacuum.

\section{Model of a Biased cMUT Membrane}

cMUTs are typically operated under a stress bias in order to obtain better electromechanical energy conversion. This is applied by means of a dc voltage, $V_{\mathrm{dc}}$, across the electrical terminals. This voltage level is set slightly below the collapse voltage, $V_{\text {collapse }}$,

$$
V_{\mathrm{dc}}=\gamma V_{\text {collapse }} \approx \gamma\left[0.7 \sqrt{\frac{512}{9}\left(\frac{c}{l_{t}^{2}}\right) \frac{\rho}{\varepsilon_{0}} \frac{l_{t}^{3} d_{o}^{3}}{a^{4}}}\right], \quad \begin{gathered}
\gamma<1, \\
(16)
\end{gathered}
$$

where $d_{o}$ is the effective gap height [33]. The generated force which attracts and bends the membrane toward the substrate is related to this voltage approximately as [15]

$$
F_{\mathrm{dc}} \approx \frac{\varepsilon_{0} S}{2 d_{o}^{2}} V_{\mathrm{dc}}^{2} .
$$

Applying this force induces a bending stress in the membrane as it is deflected. The resonance frequencies are 
affected by the presence of this induced stress. Mason's impedance expression considers the effect of residual stress in clamped membranes, which are made of very light and compliant materials. In that case the residual stress overwhelms the stiffness of the membrane and Mason's expression provides a clear indication for resonance frequencies and other parameters required for modeling. A uniformly distributed residual stress is assumed in Mason's model, and its effect is considered as an increase in Young's modulus. This may accurately represent the stress produced in cMUT membranes during manufacturing. The stress induced by biasing, however, is not uniform across the membrane and must be modeled accordingly.

We performed FEM simulations for circular silicon nitride membranes with different $a / l_{t}$ and $a / d_{o}$ ratios for different bias conditions and found the respective mechanical impedances. An iterative approach is followed to find the membrane deflection and the deflecting force distribution under static conditions [22]. In this procedure, we first applied dc bias to the un-deflected membrane and calculated the electrostatic forces. These forces are then applied to the membrane, which results in the membrane deflection. Since the electrostatic forces in the deflected membrane are different, these forces are recalculated and applied to the membrane again. We continued the iteration until the membrane deflection converged under static conditions. At this point, static deflecting force distribution is obtained. We used this distribution to pre-stress the membrane and then superimposed a uniformly distributed small signal sinusoidal driving force upon the membrane to find the mechanical impedance. The mechanical impedance is calculated as the ratio of the ac force phasor applied to the membrane divided by the resulting average velocity phasor.

We calculated the normalized mechanical impedance parameters for $a / l_{t}$ ratios ranging between 10 and 160 , $a / d_{o}$ ratios ranging between 13.3 and 160 , while bias voltage is varied between 0 and $99 \%$ of the collapse voltage for each membrane. We found the collapse voltage by simulation for each $a / l_{t}$ and $a / d_{o}$ combination. We observed that the collapse voltage levels obtained in finite element simulations are $1 \%$ to $3 \%$ lower than the values obtained from the approximate expression given in (16). We calculated the normalized model parameters again using the procedure given in Appendix A and compared the model impedances and those obtained by FEM simulation. We observed excellent agreement up to the third parallel resonance frequency, as in the case of an unbiased membrane.

The deviation in model parameters from their unbiased values depends only on the effective gap heightto-thickness ratio, $d_{o} / l_{t}$, and on the applied voltage-tocollapse voltage ratio, $\gamma$, in a very predictable manner. This deviation is observed only in thin membranes with large gap height. The model parameters are essentially unchanged otherwise. For example, any level of bias cannot induce any observable change in any of the parameters, except $C_{1}$, for a $d_{o} / l_{t}$ ratio less than 0.5 . The deviation in $C_{1}$ is also very small, less than $3 \%$ at $95 \%$ bias. This
TABLE II

VARiation of $C_{1 \text { Biased }} / C_{1 \text { UnBiased }}$.

\begin{tabular}{cccccccc}
\hline & & & & $\gamma$ & & & \\
$d_{0} / l_{t}$ & 0.4 & 0.5 & 0.6 & 0.7 & 0.8 & 0.9 & 0.95 \\
\hline$<0.25$ & 1.000 & 1.000 & 1.000 & 1.000 & 1.000 & 1.000 & 1.000 \\
0.25 & 1.000 & 1.000 & 1.000 & 1.000 & 1.000 & 0.996 & 0.994 \\
0.5 & 1.000 & 1.000 & 1.000 & 1.000 & 0.993 & 0.985 & 0.978 \\
0.75 & 1.000 & 1.000 & 1.000 & 0.993 & 0.979 & 0.961 & 0.945 \\
1 & 1.000 & 1.000 & 1.000 & 0.983 & 0.968 & 0.939 & 0.904 \\
15 & 1.000 & 0.994 & 0.990 & 0.962 & 0.930 & 0.862 & 0.806 \\
2 & 0.995 & 0.989 & 0.979 & 0.936 & 0.887 & 0.793 & 0.725 \\
3 & 0.989 & 0.973 & 0.947 & 0.875 & 0.784 & 0.655 & 0.557 \\
4 & 0.980 & 0.952 & 0.903 & 0.805 & 0.691 & 0.540 & 0.435 \\
6 & 0.956 & 0.894 & 0.795 & 0.659 & 0.508 & 0.358 & 0.281 \\
\hline
\end{tabular}

TABLE III

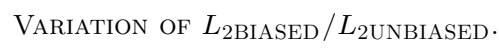

\begin{tabular}{cccccccc}
\hline & & & \multicolumn{7}{c}{$\gamma$} \\
$d_{0} / l_{t}$ & 0.4 & 0.5 & 0.6 & 0.7 & 0.8 & 0.9 & 0.95 \\
\hline$<0.75$ & 1.000 & 1.000 & 1.000 & 1.000 & 1.000 & 1.000 & 1.000 \\
0.75 & 1.000 & 1.000 & 1.000 & 1.000 & 1.000 & 1.000 & 0.992 \\
1 & 1.000 & 1.000 & 1.000 & 1.000 & 0.994 & 0.990 & 0.980 \\
1.5 & 1.000 & 1.000 & 1.000 & 0.995 & 0.985 & 0.965 & 0.941 \\
2 & 1.000 & 1.000 & 1.000 & 0.987 & 0.967 & 0.930 & 0.891 \\
3 & 1.000 & 0.995 & 0.988 & 0.964 & 0.925 & 0.854 & 0.778 \\
4 & 1.000 & 0.988 & 0.972 & 0.934 & 0.872 & 0.766 & 0.667 \\
6 & 0.992 & 0.966 & 0.928 & 0.854 & 0.744 & 0.577 & 0.465 \\
\hline
\end{tabular}

is an expected result in a relatively thick membrane made of a stiff material. The stiffening caused by the induced stress remains negligible compared to the natural stiffness of the material even under strong biasing conditions. Transducers designed for immersed operation fall into this region, because the collapse voltage is relatively low when $d_{o} / l_{t}<0.5$. On the other hand, thin membranes with large gap height may be of interest in airborne applications.

$L_{1}$ remains unchanged under all conditions. We observed that most pronounced change occurs in $C_{1}$ compared to other parameters. $C_{1}$ becomes stiffer as the $d_{o} / l_{t}$ ratio and $\gamma$ are increased. The change in the ratio of $C_{1}$ for a biased membrane to its unbiased value is given in Table II. The bias level, $\gamma$, in the table is taken as the ratio of the applied bias voltage to the approximate collapse voltage given in (16) rather than the one calculated in simulations, in order to facilitate the direct calculation of $C_{1}$. The table allows interpolation for $C_{1}$ within $1 \%$ accuracy.

$L_{2}$ is less affected. The decrease in $L_{2}$ compared to that in an unbiased membrane is similarly depicted in Table III.

Other normalized model parameters are affected by bias to lesser extent. This effect is depicted in Table IV in Appendix B. Any effect becomes observable in these parameters when the membranes have high $d_{o} / l_{t}$ ratios and they are strongly biased. Such membranes are usually useful in airborne applications for transmission. The acoustic loading presented to the membrane is low in air and the operation bandwidth is limited to a small range in the vicinity of series resonance frequency. In this frequency range, the 
effects of these model parameters are negligible and can be ignored.

We investigated the variation in the model parameters of biased membranes, when the materials are different. We simulated similar membranes $\left(a / l_{t}=80\right.$ and $\left.a / d_{o}=26.7\right)$ made of silicon and silicon carbide biased at $90 \%$ of the collapse voltage. The differences between normalized parameters obtained from membranes made of either one of the materials were negligible.

The unbiased model parameters provide an accurate representation for biased cMUT membranes. The effects of bias induced stress in the membrane are included in the normalized parameters. This allows us to use the parameter " $c$ " as it is defined in Mason's model during denormalization.

\section{iII. Parametric Model of a Clamped Membrane as a Multiport Device}

A cMUT membrane has two opposite surfaces (faces) which are in contact with the respective media. Any acoustic model of cMUT must have two symmetric acoustic ports. Driving force is applied between the back electrode surface and the membrane (e.g., negative for the inner surface-facing bottom electrode and positive for the outer surface). The model given in Fig. 1 is the model of a membrane in vacuum. The model is rearranged as in Fig. 6(b) to accommodate an input port and two sets of symmetric acoustic ports, without affecting its fundamental morphology in Fig. 1. The sense of particle velocity and force variables at every port is chosen as shown in the physical picture in Fig. 6(a).

The direction of both the force and the particle velocity on each face is chosen outward from the membrane. The reaction forces effective on both surfaces, $F_{f i}$ and $F_{b i}$, are zero, because of the vacuum termination. This rearrangement implies infinitely many acoustic ports, although there are only two faces on the membrane.

These extra ports emerge, because Mason's equation, or equivalently, the way we obtain impedance from simulation results has an implicit fundamental assumption. Defining the velocity component used in impedance calculation as the average velocity is equivalent to assuming that the impedance is the impedance of a rigid disc. We are considering the velocity of the rigid piston only. As long as the frequency is very low compared to the first parallel resonance, in vacuum any model parameters other than $L_{1}, C_{1}$, and $L_{2}$ have minimal or no effect on the vibration of the membrane. The series circuit formed by these three elements constitutes the rigid piston motion path. This path is between ports f1 and b1 via input port and connects the mechanical terminals to two acoustic ports, f1 and b1. The acoustic ports correspond to two surfaces of the membrane.

The membrane surface becomes corrugated when it is driven at higher frequencies. Parallel resonances appear as the frequency increases. Although the average velocity

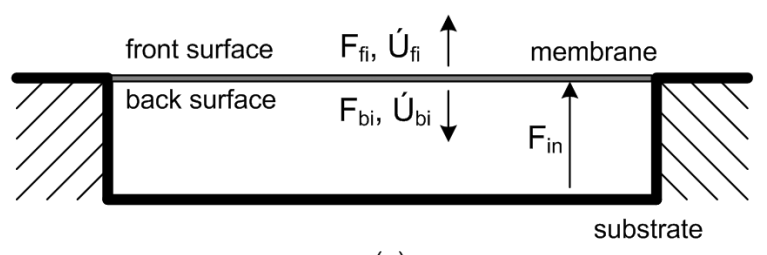

(a)

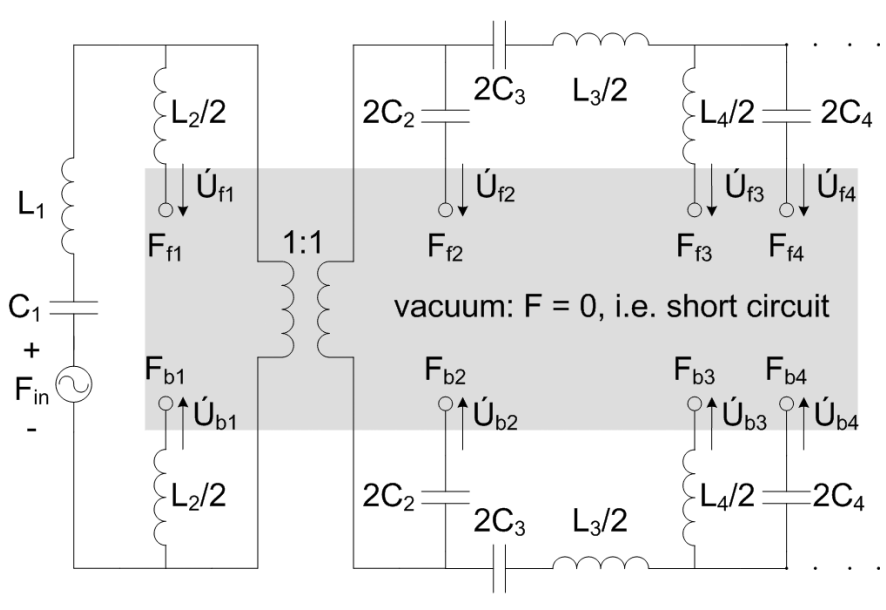

(b)

Fig. 6. (a) Physical picture of a membrane with acoustic ports, and (b) the model arranged with input port and two sets of acoustic ports.

on the surface becomes zero, and consequently impedance reaches a maximum, the particle velocity components distributed on the surface are not zero and carry energy. This energy is taken away from the disc motion path. When the membrane is immersed in a fluid, the acoustic loading on the surface of the membrane (or both surfaces) affects both the corrugation formation at the surface and all natural resonance frequencies. In order to understand the interaction of the membrane mechanics and acoustic media, all of these extra acoustic ports, f2, b2, f3, b3, etc., must be maintained in the model.

We included a 1:1 ideal transformer between the piston path and the rest of the model and associated ports, which accounts for surface corrugations, as shown in Fig. 6(b). Total particle velocity (total flow) in all of the ports, f2, $\mathrm{f} 3, \ldots$, which represent surface corrugation on front surface, must be equal to the total corrugation velocity on the back surface ports b2, b3, ... The transformer maintains this continuity condition. It is necessary in order to avoid direct and independent connection between the acoustic media and driving input through any one of these ports individually. This part of the circuit is energized by the rigid piston motion but affected by loading indirectly. Inclusion of this ideal transformer with 1:1 turns ratio does not change the basic model morphology of Fig. 1.

The model in Fig. 6(b) is a parametric model of a clamped membrane which characterizes the membrane mechanics in vacuum and where all mechanic and acoustic ports are defined. The membrane model remains unvaried when immersed. The model is tested for $a / l_{t}$ ratios larger 


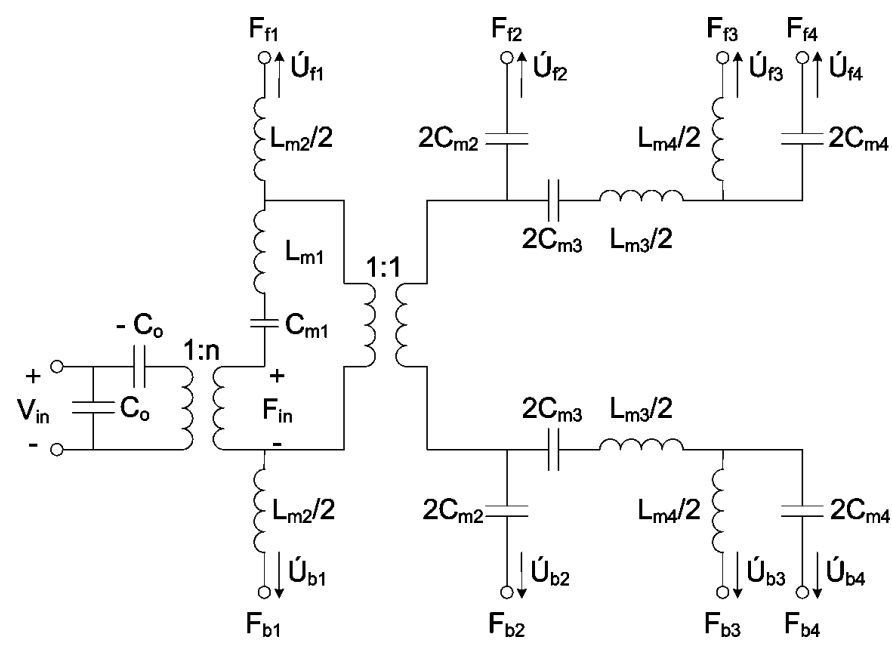

Fig. 7. Parametric model of a cMUT.

than 5 . The parameter generating polynomials in (15) is also valid for $a / l_{t}>5$. The model parameters are determined from the vibration properties in vacuum only.

The cMUT model, complete with the approximate electromechanical coupling part, is shown in Fig. 7. The model can be connected to different acoustic terminations at two different surfaces. We confined the model order to four since a very good representation is obtained in a large frequency range containing the range of interest.

The mechanical parameters in the model in Fig. 7 are fully assessed in this paper. Studies on accurate modeling of the electrical clamp capacitance $C_{0}$ reveals that increasing bias voltage increases its value slightly. When the membrane is biased near collapse, the value of $C_{0}$ is increased by about $10 \%$ at maximum, compared to its unbiased value. The accurate determination of the turns ratio, $n$, is also very important and is not studied in this work. The negative series capacitance, which represents the spring softening effect, is also known to deviate from $-C_{0}$. Various approximations for these three parameters are reported in many references for different operating conditions, which can be used in this model. A reliable estimate of these parameters will complement the accuracy in the mechanical sections.

FEM simulations are necessary in order to model the clamp capacitance, turns ratio, and the spring softening (a series negative capacitance) under different bias conditions. A complete electromechanical cMUT model can then be obtained. This is possible and requires further research. The interim results of a work to determine the values of the turns ratio and the clamp capacitance as a function of the ratio of bias voltage to the collapse voltage, using simulation results in conjunction with the membrane model parameters, are reported in [34].

\section{Application of the Model}

To gain physical insight into the model, we provide an example. Let us consider a silicon nitride cMUT membrane



Fig. 8. Comparison of the input impedance of the model and FEM simulation result for a silicon nitride membrane with a 20 -micron radius and 0.33 -micron thickness, which has a gap height of 0.6 micron and biased at $90 \%$ of collapse voltage.

with an $a / l_{t}$ of 60 and an effective gap height $d_{0}$ of $0.6 \mathrm{mi}-$ cron biased at $90 \%$ of $V_{\text {collapse }}$, which has a 20 -micron radius. Using the formulas in (15) together with Table I, we determine the normalized model parameter values for the unbiased membrane first. Then we apply the correction to $C_{1}$ and $L_{2}$ for bias using Tables II and III to obtain the normalized parameters of this membrane as $C_{1}=0.00427$, $L_{1}=1.201, C_{2}=0.000332, L_{2}=0.566, C_{3}=0.000643$, $L_{3}=0.311, C_{4}=0.000806$, and $L_{4}=0.154$. The actual values of inductors and capacitors, $C_{m k}$ and $L_{m k}$ for $k=1,2,3,4$, are found from (13) and (14) by substituting the properties of silicon nitride and the dimensions of the membrane into these equations. The input impedance of the model is plotted together with impedance obtained by FEM simulation in Fig. 8. There is very good agreement between the two impedances up to the third parallel resonance frequency, although none of the parameters except $C_{1}$ and $L_{2}$ are corrected for bias.

Modeling the interaction of the membrane with the acoustic medium accurately also requires further research. We reported the initial results of a research on this matter in [35], where the membrane model presented in this paper is employed. Preliminary results show very good agreement with measurements.

\section{Discussion and Conclusions}

A parametric modeling methodology for the mechanical impedance of a circular cMUT membrane is developed and presented. We showed that a lumped element model predicts the membrane dynamics very accurately when operated in vacuum. The modeling approach involves a novel normalization method, which strips the normalized model parameters from membrane material properties. The mechanical impedance of biased membranes 
obtained by means of FEM simulations for different radiusto-thickness ratios and different radius-to-gap height ratios are used to derive the normalized parameters as a function of these ratios. The modeling method employs the first series resonance frequency and the derivative of the mechanical reactance at this frequency, and parallel resonances and the derivatives of the susceptance at these resonances.

The frequency range in which the model is valid depends on the order of the model. As the order increases, more lumped elements are needed and the cMUT is modeled very accurately over a wider range. The model order increases by one additional $L C$ section for every successive resonance considered, and the validity range is up to the frequency of the highest resonance considered. It is more important to have a model that represents the membrane behavior accurately in the frequency range of interest. The model parameter generation polynomials are given in (15). The parameters obtained from these polynomials are accurate approximations for $a / l_{t}>5$.

We also investigated the normalized model parameters of biased membranes. We demonstrated that the parameter values depend only on the gap height-to-thickness and applied voltage-to-collapse voltage ratios. We found out that the model parameters remain unchanged for any bias level for cMUTs designed for immersion, which normally have a low gap height-to-thickness ratio. Interpolation tables are provided for determination of parameter values within $1 \%$ accuracy up to $95 \%$ bias and effective gap height-to-thickness ratio of 6 .

The full cMUT parametric model, with an approximate electromechanical coupling model, is given in Fig. 7. It has one electrical port and two sets of acoustic ports, which correspond to the two opposite faces of the membrane. Available approximations for the clamp capacitance, $C_{0}$, spring softening negative series capacitance, and the turns ratio, $n$, can be used in the model. The model can be coupled to any acoustic termination on either port.

\section{Appendix A}

The parallel resonances of the circuit in Fig. 2(b) are independent of the series branch components, $C_{1}$ and $L_{1}$. This enables us to obtain the parallel branch element values independently.

The susceptances $B_{2}$ and $B_{1}$ in Fig. 2(b) are rational polynomials of radial frequency:

$$
\begin{aligned}
j B_{2} & =\frac{j \omega C_{3}\left(1-\omega^{2} / \omega_{4}^{2}\right)}{1-\omega^{2} a+\omega^{4} b}, \\
j B_{1} & =\left(j \omega C_{2}+\frac{1}{j \omega L_{2}}\right)+j B_{2} \\
& =\frac{1-\omega^{2} c+\omega^{4} d-\omega^{6} e}{j \omega L_{2}\left(1-\omega^{2} a+\omega^{4} b\right)},
\end{aligned}
$$

where the polynomial coefficients are functions of the circuit parameters and

$$
\omega_{4}=1 / \sqrt{L_{4} C_{4}}
$$

At each parallel resonance frequency, $B_{1}$ is zero. If, for example, the first three successive resonances are considered, there are three independent equations at these resonance frequencies, $\omega_{p 1}, \omega_{p 2}$, and $\omega_{p 3}$, respectively. The radial frequency is known in each of these equations. We first determine the coefficients of the rational polynomial and then find the model parameters. The three resonance equations are as follows:

$$
\left[\begin{array}{lll}
1-\omega_{p 1}^{2} & \omega_{p 1}^{4} \\
1-\omega_{p 2}^{2} & \omega_{p 2}^{4} \\
1-\omega_{p 3}^{2} & \omega_{p 3}^{4}
\end{array}\right]\left[\begin{array}{l}
c \\
d \\
e
\end{array}\right]=\left[\begin{array}{l}
1 / \omega_{p 1}^{2} \\
1 / \omega_{p 2}^{2} \\
1 / \omega_{p 3}^{2}
\end{array}\right] .
$$

As the order increases, higher powers of $\omega_{p i}$ are used in (22), and conditioning the coefficient matrix must be considered in order to avoid ill matrix formation, while obtaining $c, d$, and $e$.

We can express the value of the derivative of $B_{1}$ at each parallel resonance frequency as

$$
\begin{aligned}
\left.\frac{d B_{1}}{d \omega}\right|_{\omega_{p i}} & =\left.\frac{d}{d \omega}\left(-\frac{1-\omega^{2} c+\omega^{4} d-\omega^{6} e}{\omega L_{2}\left(1-\omega^{2} a+\omega^{4} b\right)}\right)\right|_{\omega_{p i}} \\
& =\left.\frac{2 c-4 \omega^{2} d+6 \omega^{4} e}{L_{2}\left(1-\omega^{2} a+\omega^{4} b\right)}\right|_{\omega_{p i}}=\xi_{i},
\end{aligned}
$$

which can be put into the following form to solve for $a, b$, and $L_{2}$ :

$$
\left[\begin{array}{ccc}
1 & -\omega_{p 1}^{2} & \omega_{p 1}^{4} \\
1 & -\omega_{p 2}^{2} & \omega_{p 2}^{4} \\
1 & -\omega_{p 3}^{2} & \omega_{p 3}^{4}
\end{array}\right]\left[\begin{array}{c}
L_{2} \\
L_{2} a \\
L_{2} b
\end{array}\right]=\left[\begin{array}{l}
\left(2 c-4 \omega_{p 1}^{2} d+6 \omega_{p 1}^{4} e\right) / \xi_{1} \\
\left(2 c-4 \omega_{p 2}^{2} d+6 \omega_{p 2}^{4} e\right) / \xi_{2} \\
\left(2 c-4 \omega_{p 3}^{2} d+6 \omega_{p 3}^{4} e\right) / \xi_{3}
\end{array}\right] .(22)
$$

The components of the parallel branch are related to $a$, $b, c, d, e$, and $L_{2}$ as

$$
\begin{aligned}
C_{2} & =\frac{1}{\omega_{2}^{2} L_{2}}, \quad \text { where } \quad \frac{1}{\omega_{2}^{2}}=\frac{e}{b}, \\
C_{3} & =\frac{1}{L_{2}}\left(c-a-\frac{e}{b}\right), \\
\frac{1}{\omega_{4}^{2}} & =\frac{1}{L_{2} C_{3}}\left(d-b-a \frac{e}{b}\right) \quad \text { and } \quad \frac{1}{\omega_{3}^{2}}=b \omega_{4}^{2}, \\
L_{3} & =\frac{1}{\omega_{3}^{2} C_{3}}, \\
L_{4} & =\frac{1}{C_{3}}\left(a-\frac{1}{\omega_{3}^{2}}-\frac{1}{\omega_{4}^{2}}\right) \quad \text { and } \quad C_{4}=\frac{1}{\omega_{4}^{2} L_{4}} .
\end{aligned}
$$

The total reactance of the series branch is zero at the series resonance frequency, $\omega_{s}$, yielding

$$
\frac{-1}{\omega_{s} C_{1}}+\omega_{s} L_{1}-\frac{1}{B_{1}\left(\omega_{s}\right)}=0
$$

Also, the derivative of the total reactance at series resonance is $\beta$, hence we have,

$$
\frac{1}{\omega_{s}^{2} C_{1}}+L_{1}-\left.\frac{d}{d \omega}\left(\frac{1}{B_{1}}\right)\right|_{\omega_{s}}=\beta
$$

We solve (26) and (27) to obtain $C_{1}$ and $L_{1}$. 
TABLE IV

Variation of Normalized Parameters for Biased Membranes.

\begin{tabular}{cccccc}
\hline \multicolumn{6}{c}{$C_{2 \text { BIASED }} / C_{2 \text { UNBIASED }}$} \\
\hline$d_{0} / l_{t}$ & 0.6 & 0.7 & 0.8 & 0.9 & 0.95 \\
\hline$<3$ & 1.00 & 1.00 & 1.00 & 1.00 & 1.00 \\
3 & 1.00 & 1.00 & 1.00 & 1.02 & 1.04 \\
4 & 1.00 & 1.00 & 1.03 & 1.05 & 1.08 \\
6 & 1.01 & 1.02 & 1.05 & 1.09 & 1.14 \\
\hline
\end{tabular}

(a)

\begin{tabular}{|c|c|c|c|c|c|c|c|c|}
\hline \multicolumn{5}{|c|}{$C_{3 \text { BIASED }} / C_{3 \text { UNBIASED }}$} & \multicolumn{4}{|c|}{$L_{3 \text { BIASED }} / L_{3 \text { UNBIASED }}$} \\
\hline$d_{0} / l_{t}$ & 0.7 & 0.8 & 0.9 & 0.95 & 0.7 & 0.8 & 0.9 & 0.95 \\
\hline$<4$ & 1.00 & 1.00 & 1.00 & 1.00 & 1.00 & 1.00 & 1.00 & 1.00 \\
\hline 4 & 1.00 & 1.00 & 0.99 & 0.96 & 1.00 & 1.00 & 1.00 & 1.01 \\
\hline 6 & 1.00 & 1.00 & 0.93 & 0.78 & 1.00 & 1.02 & 1.04 & 1.11 \\
\hline \multicolumn{5}{|c|}{ (b) } & \multicolumn{4}{|c|}{ (c) } \\
\hline \multicolumn{4}{|c|}{$C_{4 \mathrm{BIASED}} / C_{4 \mathrm{UNBIASED}}$} & \multicolumn{5}{|c|}{$L_{4 \mathrm{BIASED}} / L_{4 \mathrm{UNBIASED}}$} \\
\hline$d_{0} / l_{t}$ & 0.8 & $\begin{array}{c}\gamma \\
0.9\end{array}$ & 0.95 & 0.6 & 0.7 & $\begin{array}{c}\gamma \\
0.8\end{array}$ & 0.9 & 0.95 \\
\hline$<2$ & 1.00 & 1.00 & 1.00 & 1.00 & 1.00 & 1.00 & 1.00 & 1.00 \\
\hline 2 & 1.00 & 1.00 & 0.99 & 1.00 & 1.00 & 1.00 & 1.02 & 1.03 \\
\hline 3 & 1.00 & 0.99 & 0.98 & 1.00 & 1.00 & 1.02 & 1.04 & 1.08 \\
\hline 4 & 1.00 & 0.97 & 0.95 & 1.00 & 1.02 & 1.03 & 1.08 & 1.17 \\
\hline 6 & 0.97 & 0.89 & 0.83 & 1.01 & 1.04 & 1.06 & 1.20 & 1.41 \\
\hline \multicolumn{4}{|c|}{ (d) } & & & (e) & & \\
\hline
\end{tabular}

\section{Appendix B}

The variation of normalized model parameters $C_{2}, C_{3}$, $L_{3}, C_{4}$, and $L_{4}$ with respect to $d_{o} / l_{t}$ and $\gamma$ for biased membranes are given in Table IV.

\section{REFERENCES}

[1] A. Ballato, "Modeling piezoelectric and piezomagnetic devices and structures via equivalent networks," IEEE Trans. Ultrason., Ferroelect., Freq. Contr., vol. 48, no. 5, pp. 1189-1240, 2001.

[2] W. Mason, Electromechanical Transducers and Wave Filters. 2nd ed. New York: Van Nostrand, 1948.

[3] F. V. Hunt, Electroacoustics. Cambridge, MA: Harvard University, 1954.

[4] I. Ladabaum, B. T. Khuri-Yakub, D. Spoliansky, and M. T. Haller, "Micromachined ultrasonic transducers (MUTs)," in Proc. IEEE Ultrason. Symp., 1995, pp. 501-504.

[5] M. I. Haller and B. T. Khuri-Yakub, "A surface micromachined electrostatic ultrasonic air transducer," IEEE Trans. Ultrason., Ferroelect., Freq. Contr., vol. 43, pp. 1-6, 1996.

[6] Y. Roh and B. T. Khuri-Yakub, "Finite element analysis of underwater capacitor micromachined ultrasonic transducers," IEEE Trans. Ultrason., Ferroelect., Freq. Contr., vol. 49, pp. 293-298, 2002.

[7] B. Bayram, E. Hæggström, A. S. Ergun, G. G. Yaralioglu, and B. T. Khuri-Yakub, "Dynamic analysis of cMUTs in different regimes of operation," in Proc. IEEE Ultrason. Symp., 2003, pp. 481-484.

[8] D. W. Greve, J. J. Neumann, I. J. Oppenheim, S. P. Pessiki, and D. Ozevin, "Robust capacitive MEMS ultrasonic transducers for liquid immersion," in Proc. IEEE Ultrason. Symp., 2003, pp. $581-584$.

[9] A. Lohfink, P.-C. Eccardt, W. Benecke, and H. Meixner, "Derivation of a 1D cMUT model from FEM results for lin- ear and nonlinear equivalent circuit simulation," in Proc. IEEE Ultrason. Symp., 2003, pp. 465-468.

[10] B. Bayram, G. G. Yaralioglu, A. S. Ergun, O. Oralkan, and B. T. Khuri-Yakub, "Dynamic FEM analysis of multiple CMUT cells in immersion," in Proc. IEEE Ultrason. Symp., 2004, pp. 252255.

[11] A. Austeng, J. E. Kirkeb $\varnothing$, and S. Holm, "A flexible algorithm for layout-optimized sparse cMUT arrays," in Proc. IEEE Ultrason. Symp., 2004, pp. 1266-1269.

[12] A. Caronti, A. Savoia, G. Caliano, and M. Pappalardo, "Acoustic coupling in capacitive microfabricated ultrasonic transducers: Modeling and experiments," IEEE Trans. Ultrason., Ferroelect., Freq. Contr., vol. 52, pp. 2220-2234, 2005.

[13] Y. A. Ronnekleiv, "CMUT array modeling through free acoustic CMUT modes and analysis of the fluid CMUT interface through Fourier transform methods," IEEE Trans. Ultrason., Ferroelect., Freq. Contr., vol. 52, pp. 2173-2184, 2005.

[14] A. Ronnekleiv, I. Ladabaum, X. C. Jin, and B. T. Khuri-Yakub, "An improved circuit model of MUTs," in Proc. IEEE Ultrason. Symp., 1997, pp. 395-399.

[15] I. Ladabaum, X. Jin, H. T. Soh, A. Atalar, and B. T. KhuriYakub, "Surface micromachined capacitive ultrasonic transducers," IEEE Trans. Ultrason., Ferroelect., Freq. Contr., vol. 45, pp. 678-690, 1998.

[16] G. G. Yaralioglu, M. H. Badi, A. S. Ergun, and B. T. Khuri-Yakub, "Improved equivalent circuit and finite element method modeling of capacitive micromachined ultrasonic transducers," in Proc. IEEE Ultrason. Symp., 2003, pp. 469-472.

[17] A. Bozkurt and M. Karaman, "A lumped circuit model for the radiation impedance of a 2 D cMUT array element," in Proc. IEEE Ultrason. Symp., 2005, pp. 1929-1932.

[18] A. Nikoozadeh, B. Bayram, G. G. Yaralioglu, and B. T. KhuriYakub, "Analytical calculation of collapse voltage of cMUT membrane," in Proc. IEEE Ultrason. Symp., 2004, pp. 256-259.

[19] B. J. Kirchmayer, W. A. Moussa, and M. D. Checkel, "Finite element modeling of a capacitive micromachined ultrasonic transducer," in Proc. IEEE Ultrason. Symp., 2003, pp. 465-468.

[20] R. Lerch, M. Kaltenbacher, A. Hauck, G. Link, and M. Hofer, "Accurate modeling of cMUTs," in Proc. IEEE Ultrason. Symp., 2004, pp. 264-269.

[21] D. Certon, F. Teston, and F. Patat, "A finite difference model for cMUT devices," IEEE Trans. Ultrason., Ferroelect., Freq. Contr., vol. 52, pp. 2199-2210, 2005.

[22] A. Bozkurt, I. Ladabaum, A. Atalar, and B. T. Khuri-Yakub, "Theory and analysis of electrode size optimization for capacitive microfabricated ultrasonic transducers," IEEE Trans. Ultrason., Ferroelect., Freq. Contr., vol. 46, pp. 1364-1374, 1999.

[23] B. Bayram, G. G. Yaralioglu, A. S. Ergun, and B. T. KhuriYakub, "Influence of the electrode size and location on the performance of a cMUT," in Proc. IEEE Ultrason. Symp., 2001, pp. 949-952.

[24] G. G. Yaralioglu, A. S. Ergun, and B. T. Khuri-Yakub, "Finiteelement analysis of capacitive micromachined ultrasonic transducers," IEEE Trans. Ultrason., Ferroelect., Freq. Contr., vol. 52, pp. 2185-2198, 2005.

[25] A. Bozkurt, "Finite element modeling of cMUTs using a perfectly matched layer for fast simulation," in Proc. IEEE Ultrason. Symp., 2003, pp. 1979-1982.

[26] A. Bozkurt, F. L. Degertekin, A. Atalar, and B. T. Khuri-Yakub, "Analytic modeling of loss and cross-coupling in capacitive micromachined ultrasonic transducers," in Proc. IEEE Ultrason. Symp., 1998, pp. 1025-1028.

[27] G. G. Yaralioglu, A. S. Ergun, B. Bayram, T. Marentis, and B. T. Khuri-Yakub, "Residual stress and Young's modulus measurement of capacitive micromachined ultrasonic transducer membranes," in Proc. IEEE Ultrason. Symp., 2001, pp. 953956.

[28] S. T. Hansen, A. Turo, F. L. Degertekin, and B. T. Khuri-Yakub, "Characterization of capacitive micromachined ultrasonic transducers in air using optical measurements," in Proc. IEEE Ultrason. Symp., 2000, pp. 947-950.

[29] M. H. Badi, G. G. Yaralioglu, A. S. Ergun, Y. Huang, and B. T. Khuri-Yakub, "Improved modeling and fabrication techniques for capacitive micromachined ultrasonic Lamb wave transducers," in Proc. IEEE Ultrason. Symp., 2003, pp. 585-588. 
[30] A. Caronti, G. Caliano, A. Iula, and M. Pappalardo, "Electrical impedance mismatch in capacitive micromachined ultrasonic transducers," in Proc. IEEE Ultrason. Symp., 2000, pp. 925-930.

[31] N. Lamberti, A. Caronti, G. Caliano, A. Iula, R. Carotenuto, and M. Pappalardo, "An energetic definition of the electromechanical coupling coefficient for cMUTs," in Proc. IEEE Ultrason. Symp., 2001, pp. 895-898.

[32] G. A. Baker, Jr. and P. Graves-Morris, Pade Approximants, Part I: Basic Theory. Reading, MA: Addison-Wesley, 1981.

[33] S. Olcum, M. N. Šenlik, and A. Atalar, "Optimization of the gain-bandwidth product of capacitive micromachined ultrasonic transducers," IEEE Trans. Ultrason., Ferroelect., Freq. Contr., vol. 52, pp. 2211-2219, 2005.

[34] S. Olcum, A. Atalar, H. Köymen, and M. N. Šenlik, "Calculation of transformer ratio in Mason's equivalent circuit for cMUT's," in Proc. IEEE Ultrason. Symp., to be published.

[35] M. N. Šenlik, A. Atalar, H. Köymen, and S. Olcum, "Radiation impedance and equivalent circuit for immersed cMUT array element," in Proc. IEEE Ultrason. Symp., to be published.

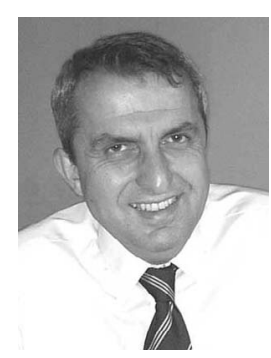

Hayrettin Köymen (M'87-SM'91) received the B.Sc. and M.Sc. degrees from Middle East Technical University (METU), Ankara, Turkey, in 1973 and 1976, respectively, and the Ph.D. degree from Birmingham University, UK, in 1979, all in electrical engineering.

He worked as a faculty member in the Marine Sciences Department (Mersin) and the Electrical Engineering Department (Ankara) of METU from 1979 to 1990, and in Bilkent University since 1990, where he is a professor. His research activities include underwater acoustic and ultrasonic transducer design, acoustic microscopy, ultrasonic NDT, biomedical instrumentation, mobile communications, and spectrum management.

Professor Köymen is a fellow of IET (formerly IEE).

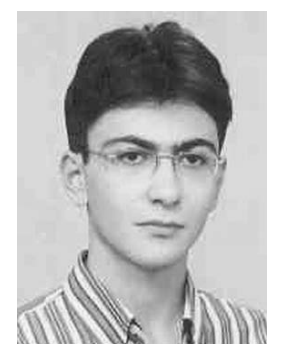

Muhammed N. Şenlik was born in Isparta, Turkey, in 1981. He received his B.S. and M.S. degrees from Bilkent University in 2002 and 2005 , respectively, both in electrical and electronics engineering. He is currently working toward his Ph.D. degree in the same department, where he has been a research assistant since 2002 .

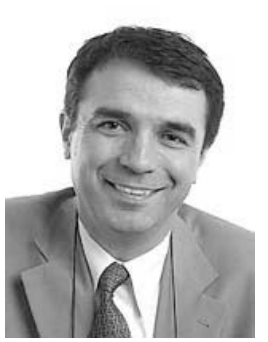

Abdullah Atalar (M'88-SM'90-F'07) received his B.S. degree from Middle East Technical University, Ankara, Turkey, in 1974, and and his M.S. and Ph.D. degrees from Stanford University, Stanford, CA, in 1976 and 1978, respectively, all in electrical engineering. From 1978 to 1980 he was first a postdoctoral fellow and later an engineering research associate in Stanford University. For 8 months he was with Hewlett Packard Labs, Palo Alto. From 1980 to 1986 he was on the faculty of the Middle East Technical University as an assistant professor. In 1986 he joined the Bilkent University as chairman of the Electrical and Electronics Engineering Department and served in the founding of the Department where he is currently a professor. He is presently the Provost of Bilkent University. During 1996-1998 he was a Visiting Professor at Stanford University. His current research interests include microwave electronics and micromachined sensors. He was awarded the Science Award of the Turkish Scientific Research Council (TUBITAK) in 1994. He is a Fellow of IEEE and a member of Turkish Academy of Sciences.

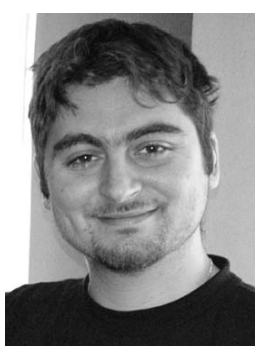

Selim Olcum was born in Chicago, IL, in 1981. He received his B.S. and M.S. degrees in electrical engineering in 2003 and 2005, respectively, both from Bilkent University.

He worked as a guest researcher at the National Institute of Standards and Technology, Semiconductor Electronics Division during the summers of 2002 and 2003. He was a visiting scholar in the Micromachined Sensors and Transducers Laboratory of Georgia Institute of Technology for 6 months in 2006. He is currently working toward his Ph.D. degree in the Department of Electrical and Electronics Engineering as Bilkent University where he has been a research assistant since 2003.

His current research interests include analysis, modeling, and optimization of cMUTs and generally MEMS.

He has been a member of the UFFC Society since 2003 . 